\title{
Dispersion relation of the fast neutrino oscillation wave
}

\author{
Changhao Yi, Lei Ma, Joshua D. Martin, and Huaiyu Duan* \\ Department of Physics \& Astronomy, University of New Mexico, \\ Albuquerque, New Mexico 87131, USA
}

(Received 7 January 2019; published 11 March 2019)

\begin{abstract}
A dense neutrino medium can support flavor oscillation waves which are coherent among different momentum modes of the neutrinos. The dispersion relation (DR) branches of such a wave with complex frequencies and/or wave numbers can lead to the exponential growth of the wave amplitude which in turn will engender a collective flavor transformation in the neutrino medium. In this work, we propose that the complex DR branches of the neutrino oscillation wave should be bound by the critical points of the DR. We demonstrate how this theory can be applied to the neutrino medium with an (approximate) axial symmetry about the propagation direction of the neutrino oscillation wave. We also show how the flavor instabilities in this medium can be identified by tracing the critical points of the DR as the electron lepton number distribution of the neutrino medium is changed continuously.
\end{abstract}

DOI: 10.1103/PhysRevD.99.063005

\section{INTRODUCTION}

Through the neutrino-neutrino forward scattering [1-3], the dense neutrino gases present in the early universe, corecollapse supernovae and binary neutron star mergers can experience collective flavor transformation (see, e.g., Refs. [4-7] among many other works, and Ref. [8] for a review). Such a collective flavor transformation can play important roles in the physical and chemical evolution of the environments of the neutrino gases. This phenomenon becomes particularly interesting after the realization that collective neutrino oscillations can occur at very high densities and on very tiny distance and time scales which are known as fast flavor conversions [9-19] (see also Ref. [20] for a review).

In the two-flavor-mixing scenario, the flavor transformation of a neutrino can be viewed as the rotation of the corresponding flavor (iso)spin $\vec{s}_{\mathbf{p}}$ in flavor space [21,22], where $\mathbf{p}$ is the momentum of the neutrino. It has been envisioned that the collective transformation of the neutrinos is engendered by the "neutrino flavor spin wave"

$$
\vec{s}_{\mathbf{p}}(t, \mathbf{r}) \propto e^{\mathrm{i}(\mathbf{K} \cdot \mathbf{r}-\Omega t)}
$$

in the neutrino medium very much like the spin wave propagating through a magnetic lattice, where $\mathbf{K}$ and $\Omega$ are

\footnotetext{
duan@unm.edu
}

Published by the American Physical Society under the terms of the Creative Commons Attribution 4.0 International license. Further distribution of this work must maintain attribution to the author(s) and the published article's title, journal citation, and DOI. Funded by SCOAP ${ }^{3}$. the wave vector and frequency of the wave, respectively [23]. However, most of the literature on collective neutrino oscillations focused on the models of only one dimension in either space or time. This paradigm was dramatically changed with the recent discoveries of the spontaneous breaking of the spatial and temporal symmetries by the collective neutrino oscillations themselves (see, e.g., Refs. [24-29], and also Ref. [30] for a review). Since then, the dispersion relation (DR) approach and the instabilities of the DR have been introduced and investigated [11-13,18].

It is clear from Eq. (1) that a DR branch with a complex $\Omega$ and/or $\mathbf{K}$ can lead to an exponential growth of the amplitude of the neutrino oscillation wave. The ranges of $\Omega$ and $\mathbf{K}$ where these complex DR branches can exist, however, is not clear. It was suggested in Ref. [11] that the complex DR branches of the neutrino oscillation wave always exist between the gaps of the real branches. This conclusion turns out to be limited to the toy model with only two neutrino beams whose DR function is a quadratic polynomial of the wave number and frequency [31]. The introduction of the general theories and classifications of the instabilities by Sturrock and Briggs in Ref. [13] was an important step forward, which shows that some of the complex-K branches actually give rise the evanescent waves instead of growing waves [32,33]. Nevertheless, the actual calculations done in Ref. [13] were still limited to the two-beam toy model. This is because the theories by Sturrock and Briggs require the knowledge of the overall analytic properties of the DR function which can be difficult to obtain for a general medium. Partly due to this difficulty, it was later proposed in Ref. [34] that the so-called "zero mode", which supposedly represents the 
overall flavor evolution of the neutrino medium, ${ }^{1}$ be used to identify the potential fast flavor conversions of the neutrinos in supernovae.

In this work, we consider the critical points of the DR function through the study of which some of the insufficiency and misunderstandings of the previous works can be addressed. The rest of the paper is organized as follows. In Sec. II, we identify the different types of the critical points of the DR function, and we propose that the complex DR branches should be bound by these critical points. In Sec. III, we illustrate this theory by several concrete examples which may be relevant to the fast flavor conversions of the supernova neutrinos in the decoupling regime. Through these examples We also demonstrate how the flavor instabilities emerge and evolve as the neutrino angular distributions of the medium changes. In Sec. IV, we give our conclusions.

\section{DISPERSION RELATION AND INSTABILITIES}

\section{A. General theories}

Although the instabilities of the normal modes have been studied extensively in other fields, it shall be helpful to recap some of the main results here. We will mostly follow Sturrock's approach [32] to the subject. To demonstrate the basic concepts of the instabilities, we consider a onedimensional medium with the wave equation

$$
\mathfrak{D}\left(\mathrm{i} \partial_{t},-\mathrm{i} \partial_{z}\right) \psi(t, z)=0,
$$

where $\psi(t, z)$ is the amplitude of the wave supported by the medium at time $t$ and position $z$, and $\mathfrak{D}\left(\mathrm{i} \partial_{t},-\mathrm{i} \partial_{z}\right)$ is a linear operator that contains the derivatives with respect to time and space. A normal mode is a solution to Eq. (2) which has the form

$$
\psi(t, z) \sim e^{\mathrm{i}(K z-\Omega t)},
$$

where $K$ and $\Omega$ are constants. Such a solution, if exists, implies the DR equation

$$
\mathfrak{D}(\Omega, K)=0 .
$$

We will use $K=\mathfrak{\Re}(\Omega)$ and $\Omega=\mathfrak{\Re}^{-1}(K)$ to denote the DR in terms of $\Omega$ and $K$, respectively.

In general, both $\Omega$ and $K$ in Eq. (4) can be complex:

$$
\Omega=\Omega_{\mathrm{r}}+\mathrm{i} \Omega_{\mathrm{i}} \quad \text { and } \quad K=K_{\mathrm{r}}+\mathrm{i} K_{\mathrm{i}} .
$$

However, in practice, one usually focuses on three kinds of DR branches ${ }^{2}$ :

\footnotetext{
${ }^{1}$ It was disclosed in the discussion part of Ref. [34] that it is possible for the system to be unstable in cases where the zero mode is stable. In other words, like any other Fourier mode, the zero mode may or may not be indicative of the overall flavor evolution of the medium.

${ }^{2}$ We use the phrase "branch" to refer to a continuous, sometimes multi-valued, DR function $\Omega(\Omega)$ for real $\Omega$ or $\Omega^{-1}(K)$ for real $K$.
}

(i) the real branches along the real axis of $\Omega$ with $\mathfrak{K}(\Omega \in \mathbb{R})$ also being real,

(ii) the complex- $K$ branches along the real axis of $\Omega$ but with $\Re(\Omega \in \mathbb{R})$ being complex,

(iii) and the complex- $\Omega$ branches along the real axis of $K$ with $\mathfrak{K}^{-1}(K \in \mathbb{R})$ being complex.

A real DR branch gives rise to propagating waves in the medium with $K$ and $\Omega$ being the wave number and the frequency of the wave, respectively. The complex- $\Omega$ and complex- $K$ branches have been called the temporal and spatial instabilities in the recent literature on neutrino oscillations because of their apparent connections to the unstable waves and the amplifying waves whose amplitudes grow in time and space, respectively. However, these apparent connections are not always true. For example, it was pointed out by Sturrock [32] that a complex- $K$ branch without a companion complex $\Omega$ branch gives rise to evanescent waves instead of amplifying waves.

To illustrate Sturrock's theory of instabilities, we consider a spatially localized perturbation at $t=0$ :

$$
\psi(0, z)=\int_{-\infty}^{\infty} \zeta(K) e^{\mathrm{i} K z} \frac{\mathrm{d} K}{2 \pi},
$$

where $\zeta(K)$ is an analytic function of $K$ that peaks at $K_{0}$ and has a finite spread. If Eq. (2) is a first-order differential equation in time, and if there exists a DR branch $\mathfrak{\Re}^{-1}(K \in \mathbb{R})$, then

$$
\psi(t, z)=\int_{-\infty}^{\infty} \zeta(K) \exp \left[\mathrm{i} K z-\mathrm{i} \mathfrak{\Re}^{-1}(K) t\right] \frac{\mathrm{d} K}{2 \pi}
$$

is the solution to Eq. (2) that satisfies the initial condition in Eq. (5). Equation (6) describes a "spacelike packet" which is bound in space at any given time. If $\mathfrak{K}^{-1}(K)$ is a real branch, the initial wave packet is transported with the group velocity

$$
V_{0}=\left(\frac{\mathrm{d} \mathfrak{\Re}^{-1}}{\mathrm{~d} K}\right)_{K=K_{0}} .
$$

If, however, (part of) $\Re^{-1}(K)$ is a complex- $\Omega$ branch, and $\operatorname{Im}\left[\Re^{-1}(K)\right]$ is positive for a range of $K$ where $|\zeta(K)|$ is appreciable, the amplitude of the wave in Eq. (6) grows exponentially in time. This indicates the presence of the temporal instabilities of the normal modes.

A temporal instability can be either convective or absolute (i.e., nonconvective). If the perturbation moves away from the point of its origin as the wave amplitude grows, the corresponding instability is convective. If, as the perturbation grows in both amplitude and extent, it still embraces the point of the origin, the instability is absolute. We illustrate the evolution of three waves with no instability, with a convective instability, and with an absolute instability in Fig. 1. Whether an instability is convective or 

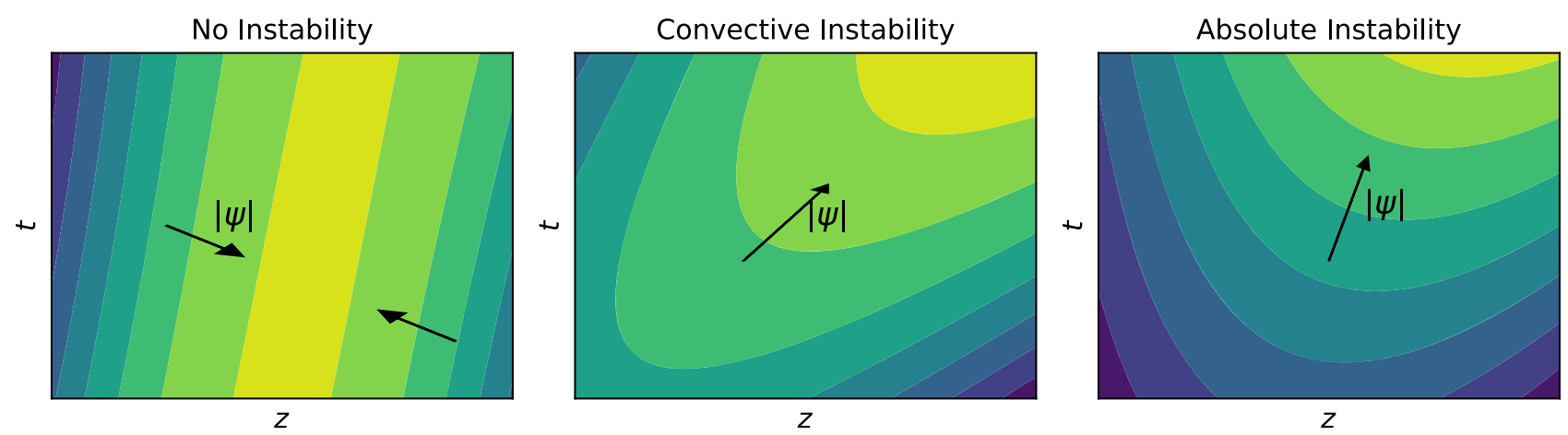

FIG. 1. The evolution of three waves with no instability (left), a convective instability (middle), and an absolute instability (right), respectively. The arrow indicates the direction of increasing wave amplitude $|\psi|$.

absolute depends on the reference frame of the observer. Nevertheless, it is useful to make this distinction because there usually is a reference frame that one prefers to work with.

Sturrock noted that, if the instability is convective, $\psi(t, z)$ is not only bound in $z$ at any given $t$ and but also bound in $t$ at any given $z$. Therefore, at any spatial point, $\psi(t, z)$ can be expressed in the form of a "timelike packet":

$$
\psi(t, z)=\int_{-\infty}^{\infty} \xi(\Omega) \exp [\mathrm{i} \Re(\Omega) z-\mathrm{i} \Omega t] \frac{\mathrm{d} \Omega}{2 \pi},
$$

where $\xi(\Omega)$ is an analytic function of $\Omega$ with a finite spread. Meanwhile, Eq. (6) can also be rewritten as

$\psi(t, z)=\int_{\mathcal{C}}\left[\zeta(\Re(\Omega)) \frac{\mathrm{d} \Omega}{\mathrm{d} \Omega}\right] \exp [\mathrm{i} \Re(\Omega) z-\mathrm{i} \Omega t] \frac{\mathrm{d} \Omega}{2 \pi}$,

where $\mathcal{C}$ is the path in the complex plane of $\Omega$ defined by $\Omega=\mathfrak{R}^{-1}(K \in \mathbb{R})$. Sturrock concluded that, if the path $\mathcal{C}$ could be continuously deformed in the complex plane of $\Omega$ to the real axis, the instability is convective, and

$$
\xi(\Omega)=\zeta(\Re(\Omega)) \frac{\mathrm{d} \Re}{\mathrm{d} \Omega} .
$$

Otherwise, the instability is absolute.

In Eq. (8), at least part of the DR branch $\Re(\Omega \in \mathbb{R})$ must be complex, or the wave will be stable. Therefore, a convective instability implies at least one companion complex- $K$ branch to the complex- $\Omega$ branch, and the presence of a complex- $\Omega$ branch alone gives rise to absolute instabilities. However, we emphasize that the above reasoning does mean that the instability is always convective when both complex- $\Omega$ and complex- $K$ branches are present. As it will be shown later, an absolute instability can exist when the complex- $\Omega$ and complex- $K$ branches do not form a closed contour on the complex plane of $\Omega$ (with the help of the real branch).

Using the symmetry between $t$ and $z$, one can easily see that the amplitude of the wave in a medium with a complex- $K$ DR branch alone will look similar to Fig. 1(c) except with $t \leftrightarrow z$. Therefore, such a complex- $K$ branch gives rise to evanescent waves which die down as $t \rightarrow \infty$ at any spatial point.

Sturrock's theory does not address the importance of the branch points of the DR function $\Re(\Omega)$. It was pointed out by Briggs [33] that a branch point $\left(\Omega_{\mathrm{b}}, K_{\mathrm{b}}\right)$ in the upper complex plane of $\Omega$ would result in absolute instabilities. In this case, the wave amplitude at a given spatial point $z$ has the following asymptotic limit

$$
\psi(t, z) \propto \frac{e^{\mathrm{i}\left(K_{\mathrm{b}} z-\Omega_{\mathrm{b}} t\right)}}{\sqrt{t}} \text { as } t \rightarrow \infty .
$$

\section{B. The dispersion relation of the fast neutrino flavor conversion}

The DR for fast neutrino flavor conversions was first derived in Ref. [11]. Here we briefly review its main results for the convenience of the readers and also to establish the formalism. We consider the mixing between two neutrino flavors, $\nu_{e}$ and $\nu_{x}$. In a dense neutrino medium with all the neutrinos initially in the weak-interaction states, the flavor content of a neutrino momentum mode $\mathbf{p}$ at time $t$ and location $\mathbf{r}$ can be described by the flavor density matrix $[11,35]$

$$
\rho_{\mathbf{p}}(t, \mathbf{r})=\frac{f_{\nu_{e}}+f_{\nu_{x}}}{2}+\frac{f_{\nu_{e}}-f_{\nu_{x}}}{2}\left[\begin{array}{cc}
s & S \\
S^{*} & -s
\end{array}\right],
$$

where $f_{\nu_{e} / \nu_{x}}(\mathbf{p})$ are the initial occupation numbers of the corresponding neutrino flavors, the real field $s_{\mathbf{p}}(t, \mathbf{r})$ and the complex field $S_{\mathbf{p}}(t, \mathbf{r})$ describe the flavor conversion and the flavor coherence of the neutrino, respectively.

In the absence of collisions, the neutrino flavor density matrix obeys the equation of motion [35]

$$
\mathrm{i}\left(\partial_{t}+\mathbf{v} \cdot \boldsymbol{\nabla}\right) \rho_{\mathbf{p}}=\left[\frac{\mathrm{M}^{2}}{2 \varepsilon}+\mathrm{H}_{\mathrm{mat}}+\mathrm{H}_{\nu \nu, \mathbf{p}}, \rho_{\mathbf{p}}\right],
$$


where $\varepsilon=|\mathbf{p}|, \mathbf{v}=\mathbf{p} / \varepsilon$ and $\mathrm{M}^{2}$ are the energy, velocity, and the mass-square matrix of the neutrino, respectively. In the above equation,

$$
\mathrm{H}_{\mathrm{mat}}=\sqrt{2} G_{\mathrm{F}} n_{e}\left[\begin{array}{ll}
1 & 0 \\
0 & 0
\end{array}\right]
$$

and

$$
\mathrm{H}_{\nu \nu, \mathbf{p}}=\sqrt{2} G_{\mathrm{F}} \int\left(1-\mathbf{v} \cdot \mathbf{v}^{\prime}\right)\left(\rho_{\mathbf{p}^{\prime}}-\bar{\rho}_{\mathbf{p}^{\prime}}\right) \frac{\mathrm{d}^{3} p^{\prime}}{(2 \pi)^{3}}
$$

are the matter and neutrino potentials, respectively, where $G_{\mathrm{F}}$ is the Fermi constant, $n_{e}$ is the electron number density, and $\bar{\rho}_{\mathbf{p}}$ is the flavor density matrix of the antineutrino which takes a form similar to Eq. (12).

We focus on fast neutrino flavor conversions which occur on very short distance and time scales over which the physical conditions such as $n_{e}$ and $f_{\nu}(\mathbf{p})\left(\nu=\nu_{e}, \bar{\nu}_{e}, \ldots\right)$ are essentially constant. The fast flavor evolution of the neutrino is energy independent because the only energydependent term $\mathrm{M}^{2} / 2 \varepsilon$ in Eq. (13) is much smaller than the rest of the Hamiltonian. (However, see Ref. [18] for a scenario where the slow and fast oscillations mix.) To be self-consistent, we also assume that no significant flavor conversion has occurred so that $\left|S_{\mathbf{v}}\right| \ll 1$ and $s_{\mathbf{v}} \approx 1$. In this case, it is useful to define the electron lepton number (ELN) distribution of the neutrino [11]

$G(\mathbf{v})=\sqrt{2} G_{\mathrm{F}} \int_{0}^{\infty}\left[\left(f_{\nu_{e}}-f_{\bar{\nu}_{e}}\right)-\left(f_{\nu_{x}}-f_{\bar{\nu}_{x}}\right)\right] \frac{\varepsilon^{2} \mathrm{~d} \varepsilon}{(2 \pi)^{3}}$,

the ELN density

$$
\Phi_{0}=\int G(\mathbf{v}) \mathrm{d} \Gamma_{\mathbf{v}}
$$

and the ELN flux density

$$
\boldsymbol{\Phi}=\int G(\mathbf{v}) \mathbf{v d} \Gamma_{\mathbf{v}},
$$

where $\mathrm{d} \Gamma_{\mathbf{v}}$ is the differential solid angle in the direction of v. Keeping only the terms of $\mathcal{O}\left(\left|S_{\mathbf{v}}\right|\right)$ or larger in Eq. (13), one obtains $[11,36]$

$$
v^{\beta}\left[\mathrm{i} \partial_{\beta}-\left(\Lambda_{\beta}+\Phi_{\beta}\right)\right] S_{\mathbf{v}}=-v^{\beta} \int v_{\beta}^{\prime} G\left(\mathbf{v}^{\prime}\right) S_{\mathbf{v}^{\prime}} \mathrm{d} \Gamma_{\mathbf{v}^{\prime}},
$$

where $\mathrm{v}=[1, \mathbf{v}]$ is the four-velocity of the neutrino, and $\Lambda=\left[\sqrt{2} G_{\mathrm{F}} n_{e}, \mathbf{0}\right]$ and $\Phi=\left[\Phi_{0}, \boldsymbol{\Phi}\right]$ are the ELN fluxes carried by the charged leptons and neutrinos, respectively.

For a normal mode of collective neutrino oscillations,

$$
S_{\mathbf{v}}(t, \mathbf{r}) \sim e^{\mathrm{i}(\mathbf{K} \cdot \mathbf{r}-\Omega t)},
$$

where $\Omega$ and $\mathbf{K}$ are the frequency and wave vector of the normal mode, respectively, both of which are independent of $\mathbf{v}$ or the (initial) flavor of the neutrino. The mixing amplitude $S_{\mathrm{v}}$ of the normal mode grows exponentially in time if $\Omega_{\mathrm{i}}>0$, and significant flavor conversions occur when $\left|S_{\mathbf{v}}\right| \sim 1$. From Eq. (19), one sees that it is convenient to make the following shifts to the frequency and wave vector which do not affect the instabilities the normal modes:

$$
\Omega-\Lambda_{0}-\Phi_{0} \rightarrow \Omega \quad \text { and } \quad \mathbf{K}-\mathbf{\Phi}-\boldsymbol{\Lambda} \rightarrow \mathbf{K} .
$$

With these shifts, the DR for the fast flavor conversions in a neutrino medium can be written as

$$
\operatorname{det}[\Pi(\Omega, \mathbf{K})]=0,
$$

where $\Pi$ is a $4 \times 4$ matrix with elements

$$
\Pi^{\beta \gamma}=\eta^{\beta \gamma}+\int G(\mathbf{v}) \frac{v^{\beta} v^{\gamma}}{\Omega-\mathbf{K} \cdot \mathbf{v}} \mathrm{d} \Gamma_{\mathbf{v}}
$$

with $\eta=\operatorname{diag}[+1,-1,-1,-1]$ being the metric tensor of the Minkowski space. Because all the parameters and constants in the above equation are real, if $(\Omega, \mathbf{K})$ is a solution to Eq. (22), so is $\left(\Omega^{*}, \mathbf{K}^{*}\right)$, where the star indicates the complex conjugate.

\section{The limits of the complex branches}

We assume that the imaginary component of $\mathbf{K}$, if it is nonzero, is parallel or anti-parallel to its real component which we assume to be along the $z$ axis. With this assumption, Eq. (23) can be written as

$$
\Pi^{\beta \gamma}=\eta^{\beta \gamma}+\int_{-1}^{1} \frac{G^{\beta \gamma}\left(v_{z}\right)}{\Omega-K v_{z}} \mathrm{~d} v_{z}
$$

where

$$
G^{\beta \gamma}\left(v_{z}\right)=\int_{0}^{2 \pi} G(\mathbf{v}) v^{\beta} v^{\gamma} \mathrm{d} \varphi
$$

with $\varphi$ being the azimuthal angle of $\mathbf{v}$ about the $z$ axis. It has been noted [11] that a real branch does not exist in the "forbidden region" where $\Pi^{\beta \gamma}$ is undefined. In this region, the "phase velocity" of the normal mode

$$
V=\frac{\Omega}{K}
$$

is within the range of $[-1,1]$.

In this subsection, we consider the limits of the complex branches. We note that the DR function $K=\mathfrak{\Re}(\Omega)$ is

\footnotetext{
${ }^{3}$ Because of the redefinition of $\Omega$ and $\mathbf{K}$ in Eq. (21), $V$ is not the actual phase velocity of the neutrino oscillation wave.
} 
(uniquely) defined by the DR equation $\mathfrak{D}(\Omega, K)=0$ in the neighborhood of the points where $\partial \mathfrak{D} / \partial K$ is defined and is nonzero. Therefore, we expect a complex- $K$ branch to end at the critical points where $\partial \mathfrak{D} / \partial K$ is either 0 or undefined. ${ }^{4}$ There are three types of critical points for the complex- $K$ branches. At a critical point of the first type, $\partial \mathfrak{D} / \partial K=0$. Near this point, the DR equation can be written as [33]

$$
\left(\frac{\partial \mathfrak{D}}{\partial \Omega}\right)_{\mathrm{b}}\left(\Omega-\Omega_{\mathrm{b}}\right)+\frac{1}{2}\left(\frac{\partial^{2} \mathfrak{D}}{\partial K^{2}}\right)_{\mathrm{b}}\left(K-K_{\mathrm{b}}\right)^{2} \approx 0,
$$

where we use the subscript "b" to indicates this type of critical point. From the above equation, one sees that $\Omega_{\mathrm{b}}$ is a branch point of $\Omega(\Omega)$ on the complex plane of $\Omega$ around which $\Re(\Omega)$ is double valued. Because $\Omega_{\mathrm{b}}$ is real, $K_{\mathrm{b}}$ must also be real so that the branch point is uniquely defined, which in turn implies that $(\partial \mathfrak{D} / \partial \Omega)_{\mathrm{b}}$ and $\left(\partial^{2} \mathfrak{D} / \partial K^{2}\right)_{\mathrm{b}}$ are both real. From Eq. (27), one sees that $\left(\Omega_{\mathrm{b}}, K_{\mathrm{b}}\right)$ is a turning point of the real branch $\Omega=\mathfrak{K}^{-1}(K \in \mathbb{R})$ where

$$
\left.\frac{\mathrm{d} \mathfrak{\Re}^{-1}}{\mathrm{~d} K}\right|_{K=K_{\mathrm{b}}}=0,
$$

and where a conjugate pair of complex- $K$ branches connect to the real branch.

A critical point of the second type is located at a finite value of $\Omega_{\mathrm{c}}$ where the phase velocity approaches a real nonzero value $V_{\mathrm{c}}$ within the forbidden region. At this critical point $\partial \mathfrak{D} / \partial K$ is undefined. The two real values of $K_{\mathrm{c}}=\Omega_{\mathrm{c}} / V_{\mathrm{c}}$ and $V_{\mathrm{c}}$ can be solved simultaneously from Eq. (22) by using

$K \Pi^{\beta \gamma} \stackrel{V \rightarrow V_{\mathrm{c}}}{\longrightarrow} K_{\mathrm{c}} \eta^{\beta \gamma}+\mathcal{P} \int_{-1}^{1} \frac{G^{\beta \gamma}\left(v_{z}\right)}{V_{\mathrm{c}}-v_{z}} \mathrm{~d} v_{z} \pm \pi \mathrm{i} G^{\beta \gamma}\left(V_{\mathrm{c}}\right)$,

where the subscript "c" indicates the values at this critical point. Here, we have used the Sokhotski-Plemelj theorem

$$
\lim _{\epsilon \rightarrow 0^{+}} \int_{a}^{b} \frac{f(x)}{x-\mathrm{i} \epsilon} \mathrm{d} x=\mathcal{P} \int_{a}^{b} \frac{f(x)}{x-\mathrm{i} \epsilon} \mathrm{d} x+\pi \mathrm{i} f(0)
$$

with the symbol $\mathcal{P}$ denoting the principal value of the integral. The critical point $\Omega_{\mathrm{c}}$ is also a branch point of $\mathfrak{K}(\Omega)$ on the complex plane of $\Omega$ where a conjugate pair of complex- $K$ branches meet. A special case of the critical points of the second type is where $V_{\mathrm{c}}$ is a crossing point of the ELN distribution so that $G\left(V_{\mathrm{c}}, \varphi\right)=0$ for all $\varphi$. In this case, $G^{\beta \gamma}\left(V_{\mathrm{c}}\right)=0$, and

\footnotetext{
${ }^{4}$ One may wonder whether a complex- $K$ branch can extend to $\Omega \rightarrow \pm \infty$. From Eq. (24), one sees that $V$ of a complex- $K$ branch must approach a finite real critical value $V_{\mathrm{c}}$ within the forbidden region as $\Omega \rightarrow \pm \infty$, or $\Pi^{\beta \gamma} \rightarrow \eta^{\beta \gamma}$ and Eq. (22) would not hold. Unless the ELN distribution is discontinuous, one can show that $V_{\text {c }}$ corresponds to a critical point of $\mathfrak{D}(\Omega, K)$.
}

$$
K \Pi^{\beta \gamma} \stackrel{V \rightarrow V_{\mathrm{c}}}{\longrightarrow} K_{\mathrm{c}} \eta^{\beta \gamma}+\mathcal{P} \int_{-1}^{1} \frac{G^{\beta \gamma}\left(v_{z}\right)}{V_{\mathrm{c}}-v_{z}} \mathrm{~d} v_{z}
$$

One can use the above expression to solve Eq. (22) for $K_{\mathrm{c}}$ and $\Omega_{\mathrm{c}}$.

The last type of the critical points is at $\Omega=0$ where $\partial \mathfrak{D} / \partial K$ is also undefined. Unlike a critical point of the second type, $K_{0}=\mathfrak{K}(0)$ is complex which can be solved from Eq. (22) by using

$$
K \Pi^{\beta \gamma} \stackrel{V \rightarrow 0}{\longrightarrow} K_{0} \eta^{\beta \gamma}-\mathcal{P} \int_{-1}^{1} \frac{G^{\beta \gamma}\left(v_{z}\right)}{v_{z}} \mathrm{~d} v_{z} \pm \pi \mathrm{i} G^{\beta \gamma}(0) .
$$

Similarly, a complex- $\Omega$ branch can end at two types of critical points. At a type-I critical point $\left(\Omega_{\mathrm{t}}, K_{\mathrm{t}}\right)$, $\partial \mathfrak{D} / \partial \Omega=0$. This point is also a turning point of the real branch $K=\mathfrak{\Re}(\Omega \in \mathbb{R})$ with

$$
\left.\frac{\mathrm{d} \Re}{\mathrm{d} \Omega}\right|_{\Omega=\Omega_{\mathrm{t}}}=0,
$$

where we use the subscript " $\mathrm{t}$ " to indicate this type of critical points. A conjugate pair of complex- $\Omega$ branches join a real branch at $\left(\Omega_{\mathrm{t}}, K_{\mathrm{t}}\right)$. A type-II critical point for the complex- $K$ branches is also a type-II critical point for the complex $\Omega$ branches where both $\partial \mathfrak{D} / \partial K$ and $\partial \mathfrak{D} / \partial \Omega$ are undefined. The points where $K=0$ are not critical because $\partial \mathfrak{D} / \partial \Omega$ is well defined there.

\section{NEUTRINO MEDIA WITH THE AXIAL SYMMETRY}

As a concrete example, we consider the neutrino media with an (approximate) axial symmetry about the $z$ axis. For such a medium, Eq. (22) gives two DRs with different symmetry properties [11]:

$$
\mathfrak{D}_{\mathrm{AS}}(\Omega, K)=\left(I_{0}+1\right)\left(I_{2}-1\right)-I_{1}^{2}=0
$$

and

$$
\mathfrak{D}_{\mathrm{SB}}(\Omega, K)=I_{0}-I_{2}-2=0
$$

where

$$
I_{k}(\Omega, K)=\int_{-1}^{1} G\left(v_{z}\right) \frac{v_{z}^{k}}{\Omega-K v_{z}} \mathrm{~d} v_{z}
$$

with

$$
G\left(v_{z}\right)=\int_{0}^{2 \pi} G(\mathbf{v}) \mathrm{d} \varphi .
$$

Equation (34) is for the normal modes of axially symmetric (AS) polarizations (i.e., $S_{\mathbf{v}}$ is independent of the azimuthal angle $\varphi$ ), while Eq. (35) is for those with (axial-)symmetrybreaking (SB) polarizations [11,37]. The AS modes are the mixtures of the monopole mode $(\ell=0)$ and the axially 
TABLE I. The parameters used in the ELN distributions in Eq. (40).

\begin{tabular}{llll}
\hline \hline$i$ & $\alpha_{i}$ & $\bar{v}_{i}$ & $\sigma_{i}$ \\
\hline 0 & $\cdots$ & 1.0 & 0.6 \\
1 & 0.88 & 1.0 & 0.53 \\
2 & 0.89 & 1.0 & 0.53 \\
3 & 0.908 & 1.0 & 0.53 \\
4 & 0.93 & 1.0 & 0.53 \\
5 & 0.96 & 1.0 & 0.53 \\
6 & 0.97 & 1.0 & 0.53 \\
\hline \hline
\end{tabular}

symmetric dipole mode $(\ell=1$ and $m=0)$, and the SB modes are the linear superposition of the two degenerate dipole modes with $m= \pm 1$ [38].

Because the typical distance scale of fast neutrino flavor conversions is $\sim G_{\mathrm{F}} n_{\nu}$, we define

$$
\mu=\sqrt{2} G_{\mathrm{F}} n_{\nu_{e}}
$$

to be the unit of all quantities with dimensions, where

$$
n_{\nu_{e}}=\int f_{\nu_{e}}(\mathbf{p}) \frac{\mathrm{d}^{3} p}{(2 \pi)^{3}}
$$

is the (initial) number density of $\nu_{e}$ 's. To demonstrate the typical features of the DRs of fast neutrino flavor conversions, we employ a set of 6 parametrized distributions all of which are of the form

$$
G_{i}\left(v_{z}\right)=\mu\left[g\left(v_{z}, \bar{v}_{0}, \sigma_{0}\right)-\alpha_{i} g\left(v_{z}, \bar{v}_{i}, \sigma_{i}\right)\right],
$$

where $i=1,2, \ldots, 6$,

$$
g\left(v_{z}, \bar{v}, \sigma\right) \propto \exp \left[-\frac{\left(v_{z}-\bar{v}\right)^{2}}{2 \sigma^{2}}\right]
$$

is the Gaussian distribution with the normalization condition $\int_{-1}^{1} g \mathrm{~d} v_{z}=1$, and the values of $\alpha_{i}, \bar{v}_{i}$, and $\sigma_{i}$ are listed in Table I. All the distributions have positive ELN

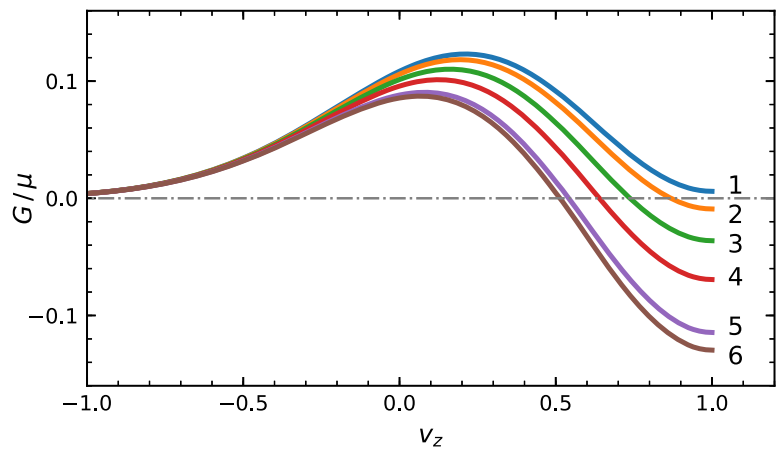

FIG. 2. The ELN distributions $G_{i}\left(v_{z}\right)(i=1,2, \ldots, 6)$ used in the numerical examples as measured by the strength of the neutrino potential $\mu=\sqrt{2} G_{\mathrm{F}} n_{\nu_{e}}$. densities $\Phi_{0}>0$, but their ELN flux densities $\Phi_{z}$ in the $z$ direction can be either positive or negative:

(i) $G_{1}$ is a distribution that stays positive for all $v_{z}$;

(ii) $G_{2}$ has a "shallow" crossing and a positive $\Phi_{z}$;

(iii) $G_{3}$ and $G_{4}$ have "moderate" crossings and negative $\Phi_{z}$;

(iv) $G_{5}$ and $G_{6}$ have "deep" crossings and negative $\Phi_{z}$. These ELN distributions are plotted in Fig. 2.

\section{A. Symmetry-breaking modes}

\section{Real branches}

The real DR branches of the SB modes can be readily solved from Eq. (35) in terms of the "refractive index" [11]

$$
\mathfrak{n}=\frac{1}{V}=\frac{K}{\Omega} .
$$

For each value of $\mathfrak{n} \in[-1,1]$, one obtains

$$
\Omega(\mathfrak{n})=\frac{1}{2} \int_{-1}^{1} G\left(v_{z}\right) \frac{1-v_{z}^{2}}{1-\mathfrak{n} v_{z}} \mathrm{~d} v_{z}
$$

and

$$
K(\mathfrak{n})=\mathfrak{n} \Omega .
$$

As noted in Ref. [11], the real branches of the SB modes always end at finite values of $\Omega$ and $K$ because

$$
\Omega(\mathfrak{n}= \pm 1)=\frac{1}{2} \int_{-1}^{1} G\left(v_{z}\right)\left(1 \pm v_{z}\right) \mathrm{d} v_{z}
$$

are finite.

As mentioned in Sec. II C, the turning points of the real branches are also critical points of the DR. To locate these turning points, we differentiate Eq. (43) with respect to $\mathfrak{n}$ and obtain

$$
\begin{aligned}
& \frac{\mathrm{d} \Omega}{\mathrm{d} \mathfrak{n}}=\frac{1}{2} \int_{-1}^{1} G\left(v_{z}\right) \frac{v_{z}\left(1-v_{z}^{2}\right)}{\left(1-\mathfrak{n} v_{z}\right)^{2}} \mathrm{~d} v_{z}, \\
& \frac{\mathrm{d}^{2} \Omega}{\mathrm{dn}^{2}}=\int_{-1}^{1} G\left(v_{z}\right) \frac{v_{z}^{2}\left(1-v_{z}^{2}\right)}{\left(1-\mathfrak{n} v_{z}\right)^{3}} \mathrm{~d} v_{z}, \\
& \frac{\mathrm{d} K}{\mathrm{~d} \mathfrak{n}}=\frac{1}{2} \int_{-1}^{1} G\left(v_{z}\right) \frac{1-v_{z}^{2}}{\left(1-\mathfrak{n} v_{z}\right)^{2}} \mathrm{~d} v_{z} .
\end{aligned}
$$

Based on the overall geometric shapes of their real DR branches, the six parametrized ELN distributions fall into three categories.

The first category of the ELN distributions includes $G_{1}$ which remains positive for the whole range of $v_{z}$. The real branch of an ELN distribution in this category has the following properties:

(i) $K(\mathfrak{n})$ is a monotonically increasing function of $\mathfrak{n}$ because $\mathrm{d} K / \mathrm{d} \mathfrak{n}>0$. 
(ii) Both $\Omega(\mathfrak{n})$ and $\mathrm{d}^{2} \Omega / \mathrm{dn}^{2}$ stay positive for the whole range of $v_{z}$, but $\mathrm{d} \Omega / \mathrm{d} \mathfrak{n}$ changes sign between $v_{z}=-1$ and 1 because

$$
\frac{\mathrm{d} \Omega}{\mathrm{d} \mathfrak{n}} \stackrel{\mathfrak{n} \rightarrow-1^{+}}{\longrightarrow} G(-1) \ln (1+\mathfrak{n})<0
$$

and

$$
\frac{\mathrm{d} \Omega}{\mathrm{d} \mathfrak{n}} \stackrel{\mathfrak{n} \rightarrow 1^{-}}{\longrightarrow}-G(1) \ln (1-\mathfrak{n})>0
$$

This implies the existence of a turning point $\left(\Omega_{\mathrm{b}}, K_{\mathrm{b}}\right)$ on the real branch where $\mathrm{d} \Omega / \mathrm{d} K=0$.

The second category includes $G_{2}$ and $G_{3}$ which are slightly negative in the forward direction $\left(v_{z}=1\right)$. The real branch of a category-II distribution is similar to that of category I but with the following important differences:

(i) $\mathrm{d} K / \mathrm{d} \mathfrak{n}$ changes sign near $\mathfrak{n}=1$ because

$$
\frac{\mathrm{d} K}{\mathrm{~d} \mathfrak{n}} \stackrel{\mathfrak{n} \rightarrow 1^{-}}{\longrightarrow}-G(1) \ln (1-\mathfrak{n})<0
$$

This implies the existence of a turning point $\left(\Omega_{\mathrm{t}}, K_{\mathrm{t}}\right)$ on the real branch where $\mathrm{d} K / \mathrm{d} \Omega=0$.

(ii) $\mathrm{d} \Omega / \mathrm{d} \mathfrak{n}$ changes sign again near $\mathfrak{n}=1$ where $\mathrm{d} \Omega / \mathrm{d} \mathfrak{n}$ becomes negative [see Eq. (47)]. This indicates the appearance of another turning point $\left(\Omega_{\mathrm{b}}^{\prime}, K_{\mathrm{b}}^{\prime}\right)$ on the real branch.

The last category of the ELN distributions include $G_{4}$ through $G_{6}$ whose real DR branches have only one critical point $\left(\Omega_{\mathrm{t}}, K_{\mathrm{t}}\right)$.

We calculated the real DR branches for $G_{1}, G_{3}$ and $G_{5}$, which represent the three categories of ELN distributions, and show them as solid curves in Fig. 3.

\section{Complex-K and complex- $\Omega$ branches}

For the SB modes, a type-II critical point $\left(\Omega_{\mathrm{c}}, K_{\mathrm{c}}\right)$ can exist only if the ELN distribution $G\left(v_{z}\right)$ has a crossing point $V_{\mathrm{c}}$. From the above discussion, one sees that, for a category-I distribution, there are only two critical points for the complex- $K$ branches, $\Omega=0$ and $\Omega_{\mathrm{b}}$. Therefore, a pair of complex- $K$ branches run from $\left(\Omega_{\mathrm{b}}, K_{\mathrm{b}}\right)$ to $\left(0, K_{0}\right)$ and $\left(0, K_{0}^{*}\right)$, respectively. For a category-II distribution, there are two additional critical points, $\Omega_{\mathrm{b}}^{\prime}$, and $\Omega_{\mathrm{c}}$. As a result, another pair of complex- $K$ branches run from $\left(\Omega_{\mathrm{c}}, K_{\mathrm{c}}\right)$ to $\left(\Omega_{\mathrm{b}}^{\prime}, K_{\mathrm{b}}^{\prime}\right)$. For a category-III distribution, there are again only two critical points, 0 and $\Omega_{\mathrm{c}}$, and a pair of complex- $K$ branches run from $\left(\Omega_{\mathrm{c}}, K_{\mathrm{c}}\right)$ to $\left(0, K_{0}\right)$ and $\left(0, K_{0}^{*}\right)$, respectively.

A category-I ELN distribution does not have any complex- $\Omega$ branch because there does not exist any critical point for these branches. Both category-II and category-III
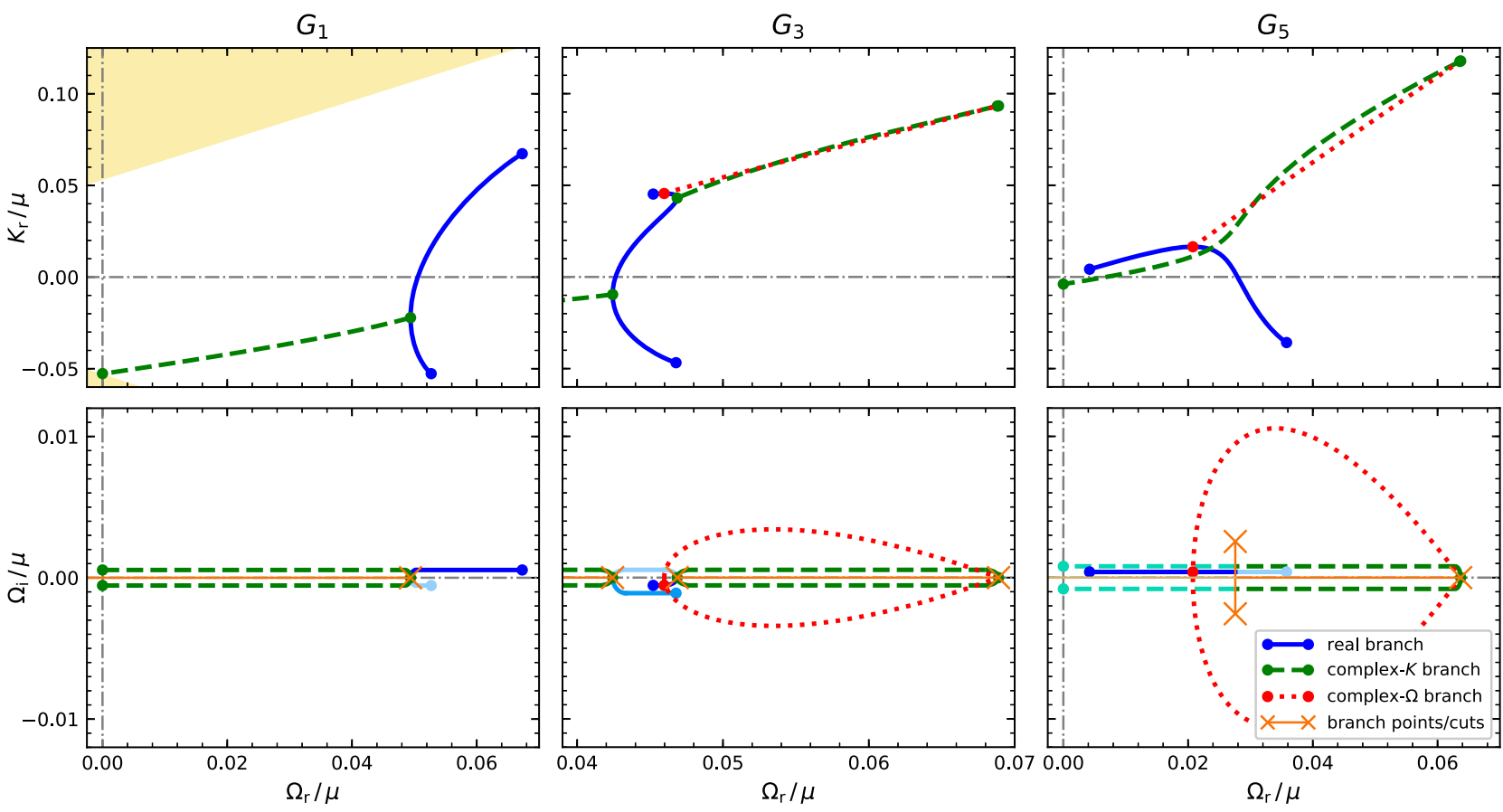

FIG. 3. The real (solid lines), complex- $K$ (dashed lines) and complex- $\Omega$ (dotted lines) DR branches for the SB modes with $G_{1}$ (left panels), $G_{3}$ (middle panels) and $G_{5}$ (right panels) distributions on the $\Omega_{\mathrm{r}}-K_{\mathrm{r}}$ plane (upper panels) and the complex plane of $\Omega$ (lower panels), respectively. The filled circles represent the end points of the branches, and the crosses denote the branch points on the complex plane of $\Omega$ where two Riemann sheets join. The shadowed regions in the upper panels are the forbidden regions for the real branches. In the lower panels, the lines of the same style but different colors/intensities represent the same DR branches on different Riemann sheets, and the DR branches along the real axis of the complex plane of $\Omega$ are shifted up or down for clarity. 
distributions have two critical points which are located at $K=K_{\mathrm{t}}$ and $K_{\mathrm{c}}$, respectively. Therefore, a pair of complex- $\Omega$ branches run from $\left(\Omega_{\mathrm{c}}, K_{\mathrm{c}}\right)$ to $\left(\Omega_{\mathrm{t}}, K_{\mathrm{t}}\right)$.

We calculated the complex- $K$ and complex- $\Omega$ branches for $G_{1}, G_{3}$ and $G_{5}$, respectively, and show them as dashed and dotted curves in Fig. 3.

\section{B. Identifying the instabilities}

The above results clearly show some of the misunderstandings in the literature. For example, instead of being confined to the "gap" of the real branches as suggested by Ref. [11], the complex- $K$ branches for the $G_{5}$ distribution co-exist with the real branch in some range of $\Omega_{\mathrm{r}}$. One also sees that the complex $-\Omega$ branches exist for this distribution even though the zero mode is stable, i.e., $\mathfrak{K}^{-1}(K=0)$ is real.

The study of the DR branches of the SB modes reveals an interesting pattern of how the instabilities appear and evolve as the ELN distribution is changed continuously. One starts from a category-I distribution without crossing which has no complex- $\Omega$ branch. For this distribution, all the branch points $\left(\Omega_{\mathrm{b}}, K_{\mathrm{b}}\right)$ are located on the real axis of the complex plane of $\Omega$, and they are the turning points on the real branch. There is no branch point on the upper or lower complex plane which would imply the existence of complex $-\Omega$ branches [33].

The instabilities may begin to occur as one varies the ELN distribution. Because the strength of the instability increases with the magnitude of $\operatorname{Im}\left(\Omega_{\mathrm{b}}\right)$, we expect that all the branch points should appear on the real axis first before moving to the upper and lower complex plane of $\Omega$. As the ELN distribution begins to develop a shallow crossing and become category II, two new turning points, $\left(\Omega_{\mathrm{b}}^{\prime}, K_{\mathrm{b}}^{\prime}\right)$ and $\left(\Omega_{\mathrm{t}}, K_{\mathrm{t}}\right)$ appear on the reach branch. The complex- $\Omega$ branch (with $\left.\Omega_{\mathrm{i}}>0\right)$ connecting to $\left(\Omega_{\mathrm{t}}, K_{\mathrm{t}}\right)$ gives rise to convective instabilities because there is no branch point on the upper complex plane of $\Omega$. This also agrees with Sturrock's theory because the path $\mathcal{C}$ of this complex- $\Omega$ branch can be deformed continuously in the complex plane of $\Omega$ to a path along the real axis which is made of a complex- $K$ branch and a segment of the real branch. (See the lower middle panel of Fig. 3).

As the crossing of a category-II distribution becomes deeper and deeper, the two turning points $\left(\Omega_{\mathrm{b}}, K_{\mathrm{b}}\right)$ and $\left(\Omega_{\mathrm{b}}^{\prime}, K_{\mathrm{b}}^{\prime}\right)$ on the real branch come closer and closer to each other and finally merge into a saddle point. Correspondingly, the two pairs of complex- $K$ branches merge into a single pair. As the crossing of the ELN distribution further deepens, the two branch points of $\Omega(\Omega)$ move to the upper and lower complex planes with $\Omega_{\mathrm{b}}^{\prime}=\Omega_{\mathrm{b}}^{*}$ and $K_{\mathrm{b}}^{\prime}=K_{\mathrm{b}}^{*}$. Although these branch points are not on the real branch, their existence can be inferred by the fact that the complex- $K$ and real branches pass each other without intersection. (See the upper right panel of Fig. 3.) According to Briggs' theory, the instabilities associated with the complex- $\Omega$ branch of a category-III distribution are absolute. This conclusion is also in agreement with Sturrock's theory because the path of the complex$\Omega$ branch in the upper complex plane of $\Omega$ cannot be deformed to the real axis due to the existence of the branch point. (See the lower right panel of Fig. 3.) This result can also be deduced from the paths of the DR branches in the $\Omega_{\mathrm{r}}-K_{\mathrm{r}}$ plane where no closed loop is formed by the complex $\Omega$ branch and other DR branches with real $\Omega$.

\section{Axially symmetric modes}

The real branches of the AS modes can also be solved in terms of $\mathfrak{n}$ :

$$
\Omega_{ \pm}(\mathfrak{n})=\frac{\tilde{I}_{2}-\tilde{I}_{0} \pm \sqrt{\Delta}}{2}
$$

where

$$
\tilde{I}_{k}(\mathfrak{n})=\int_{-1}^{1} G\left(v_{z}\right) \frac{v_{z}^{k}}{1-\mathfrak{n} v_{z}} \mathrm{~d} v_{z}
$$

and

$$
\begin{aligned}
\Delta(\mathfrak{n}) & =\left(\tilde{I}_{2}-\tilde{I}_{0}\right)^{2}+4\left(\tilde{I}_{2} \tilde{I}_{0}-\tilde{I}_{1}^{2}\right) \\
& =\left(\tilde{I}_{0}+\tilde{I}_{2}-2 \tilde{I}_{1}\right)\left(\tilde{I}_{0}+\tilde{I}_{2}+2 \tilde{I}_{1}\right) .
\end{aligned}
$$

The evolution of the DR branches of the AS modes with the changing ELN distribution is similar to that of the SB modes but with a few new twists. For the AS modes, the distributions $G_{1}$ through $G_{6}$ each has its unique features and represents its own category. We calculated the DR branches of the AS modes for all 6 distributions and plot them in Fig. 4.

\section{Category I}

The distributions represented by $G_{1}$ has no crossing. For such a distribution, $\tilde{I}_{0}>\tilde{I}_{2}>0$ and $\tilde{I}_{2} \tilde{I}_{0}>\tilde{I}_{1}^{2}$. Therefore, $\Omega_{ \pm}(\mathfrak{n})$ represent two separate real branches. In the limit $\mathfrak{n} \rightarrow \pm 1, \Omega_{ \pm} \rightarrow \pm \infty$ because

$\Delta \stackrel{\mathfrak{n} \rightarrow \pm 1}{\longrightarrow}-4 G( \pm 1) \ln (1 \mp \mathfrak{n}) \int_{-1}^{1}\left(1 \mp v_{z}\right) G\left(v_{z}\right) \mathrm{d} v_{z}$.

The "plus" and "minus" real branches each has a critical point, $\left(\Omega_{\mathrm{b}+}, K_{\mathrm{b}+}\right)$ and $\left(\Omega_{\mathrm{b}-}, K_{\mathrm{b}-}\right)$, and two conjugate pairs of complex- $K$ branches run from these critical points to $\left(0, K_{0+}\right),\left(0, K_{0+}^{*}\right),\left(0, K_{0-}\right)$, and $\left(0, K_{0-}^{*}\right)$, respectively. There is no complex- $\Omega$ branch for a category-I distribution because there exists no critical point associated with these branches.

\section{Category II}

The distributions represented by $G_{2}$ has a shallow crossing near the forward direction. Because $G(1)$ is 

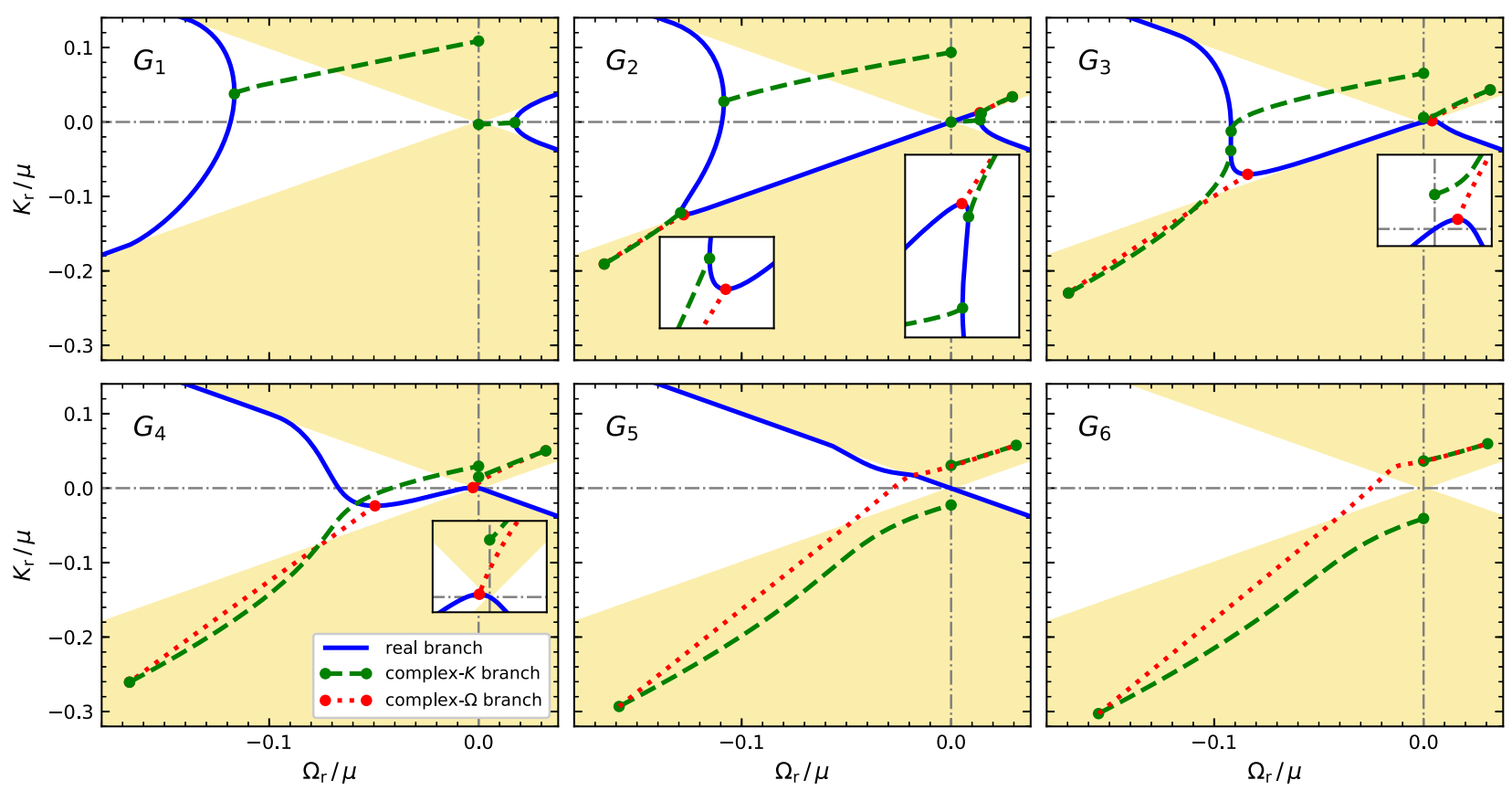

FIG. 4. Similar to Fig. 3 but for the AS modes with the ELN distributions $G_{1}$ through $G_{6}$. The small insets show the enlarged region around some of the critical points on the real DR branches.

slightly negative, $\Delta \stackrel{\mathfrak{n} \rightarrow 1^{-}}{\rightarrow}-\infty$. As a result, the real branch $\Omega_{ \pm}(\mathfrak{n})$ is defined for $\mathfrak{n} \in\left(-1, \mathfrak{n}_{\star}\right]$, where $\Delta\left(\mathfrak{n}_{\star}\right)=0$. The originally separated two real branches bend sharply toward each other near $V=1$ and merge into a single real branch at $V=1 / \mathfrak{n}_{\star}$. Around the sharp bends of the real branch new critical points $\left(\Omega_{\mathrm{b} \pm}^{\prime}, K_{\mathrm{b} \pm}^{\prime}\right)$ and $\left(\Omega_{\mathrm{t} \pm}, K_{\mathrm{t} \pm}\right)$ appear. Another pair of critical points $\left(\Omega_{\mathrm{c} \pm}, K_{\mathrm{c} \pm}\right)$ also appear on the positive and negative sides of $\Omega_{\mathrm{r}}$ both of which correspond to the crossing point $V_{\mathrm{c}}$ of the ELN distribution. With the appearance of the new critical points, two additional conjugate pairs of complex- $K$ branches run from $\left(\Omega_{\mathrm{b} \pm}^{\prime}, K_{\mathrm{b} \pm}^{\prime}\right)$ to $\left(\Omega_{\mathrm{c} \pm}, K_{\mathrm{c} \pm}\right)$, and two conjugate pairs of complex- $\Omega$ branches run from $\left(\Omega_{\mathrm{t} \pm}, K_{\mathrm{t} \pm}\right)$ to $\left(\Omega_{\mathrm{c} \pm}, K_{\mathrm{c} \pm}\right)$. Both complex $\Omega$ branches with $\Omega_{\mathrm{i}}>0$ give rise to convective instabilities.

\section{Category III}

The distributions represented by $G_{3}$ have deeper crossings than $G_{2}$. On the positive side of $\Omega_{\mathrm{r}}$, the two branch points $\left(\Omega_{\mathrm{b}+}, K_{\mathrm{b}+}\right)$ and $\left(\Omega_{\mathrm{b}+}^{\prime}, K_{\mathrm{b}+}^{\prime}\right)$ have moved off the real branch to the upper and lower complex planes of $\Omega$, and the two pairs of complex- $K$ branches have merged into a single pair. Correspondingly, the instabilities associated with the complex- $\Omega$ branch connecting to $\left(\Omega_{\mathrm{c}+}, K_{\mathrm{c}+}\right)$ become absolute.

\section{Category IV}

The distributions represented by $G_{4}$ have even deeper crossings than $G_{3}$. At this point, the two branch points $\left(\Omega_{\mathrm{b}-}, K_{\mathrm{b}_{-}}\right)$and $\left(\Omega_{\mathrm{b}-}^{\prime}, K_{\mathrm{b}-}^{\prime}\right)$ have also moved off the real branch to the upper and lower complex planes of $\Omega$, and the two pair of complex- $K$ branches on the negative side of $\Omega_{\mathrm{r}}$ have merged into a single pair. The complex $\Omega$ branches on both the positive and negative sides of $\Omega_{\mathrm{r}}$ give rise to absolute instabilities.

\section{Category V}

As the crossing of the ELN distribution becomes deeper and deeper, the two turning points $\left(\Omega_{\mathrm{t}+}, K_{\mathrm{t}+}\right)$ and $\left(\Omega_{\mathrm{t}-}, K_{\mathrm{t}-}\right)$ move closer and closer to each other and eventually merge into a saddle point before disappearing from the real branch. For the $G_{5}$ distribution, the two pairs of complex- $\Omega$ branches have merged into a single pair and run from $\left(\Omega_{\mathrm{c}+}, K_{\mathrm{c}+}\right)$ directly to $\left(\Omega_{\mathrm{c}-}, K_{\mathrm{c}-}\right)$.

\section{Category VI}

As the crossing of the ELN distribution becomes so deep that $\Phi_{0}+\Phi_{z}<0$ [but still with $G(-1)>0$ ], $\Delta$ becomes negative even at $\mathfrak{n}=-1$. At this point, the real branch has disappeared leaving only two pairs of complex- $K$ branches and one pair of complex $\Omega$ branches.

\section{CONCLUSIONS}

We have studied the critical points of the DR of the fast flavor conversion of the neutrino medium. These critical points are also the end points of the DR branches with complex frequencies and/or wave numbers. Applying this theory to the neutrino medium with the axial symmetry, we demonstrated how the DR branches and instabilities emerge and evolve as the ELN distribution is changed 
continuously. We showed that, as one starts from an ELN distribution with no unstable DR branches and varies it continuously, the branch points of $K=\mathfrak{\Re}(\Omega)$ first appear as the turning points on the real branch(es) before moving to the upper and lower complex planes of $\Omega$. In this process, convective instabilities always appear first and then may evolve into absolute instabilities as the ELN distribution changes.

We have explicitly shown that the instability associated with a complex- $\Omega$ DR branch can still be nonconvective even in the presence of complex- $K$ branches. We have also shown that fast flavor conversions can occur even when the "zero mode" is stable. Our theory of the critical points of the DR function provides a way of systematically studying the DRs and instabilities of the neutrino oscillation wave. It also contributes to a good theoretical foundation for the future studies of collective neutrino oscillations in corecollapse supernovae and binary neutrino star mergers such as those in Refs. [12,19].

\section{ACKNOWLEDGMENTS}

We thank S. Abbar for the useful discussion. We acknowledge the support by the U.S. Department of Energy NP Grant No. DE-SC0017803 at UNM.
[1] G. M. Fuller, R. W. Mayle, J. R. Wilson, and D. N. Schramm, Resonant neutrino oscillations and stellar collapse, Astrophys. J. 322, 795 (1987).

[2] D. Notzold and G. Raffelt, Neutrino dispersion at finite temperature and density, Nucl. Phys. B307, 924 (1988).

[3] J. T. Pantaleone, Dirac neutrinos in dense matter, Phys. Rev. D 46, 510 (1992).

[4] V. A. Kostelecký, J. T. Pantaleone, and S. Samuel, Neutrino oscillation in the early universe, Phys. Lett. B 315, 46 (1993).

[5] S. Pastor and G. Raffelt, Flavor Oscillations in the Supernova Hot Bubble Region: Nonlinear Effects of Neutrino Background, Phys. Rev. Lett. 89, 191101 (2002).

[6] H. Duan, G. M. Fuller, J. Carlson, and Y.-Z. Qian, Coherent Development of Neutrino Flavor in the Supernova Environment, Phys. Rev. Lett. 97, 241101 (2006).

[7] A. Malkus, J. P. Kneller, G. C. McLaughlin, and R. Surman, Neutrino oscillations above black hole accretion disks: Disks with electron-flavor emission, Phys. Rev. D 86, 085015 (2012).

[8] H. Duan, G. M. Fuller, and Y.-Z. Qian, Collective neutrino oscillations, Annu. Rev. Nucl. Part. Sci. 60, 569 (2010).

[9] R. F. Sawyer, Neutrino Cloud Instabilities Just Above the Neutrino Sphere of a Supernova, Phys. Rev. Lett. 116, 081101 (2016).

[10] S. Chakraborty, R. Sloth Hansen, I. Izaguirre, and G. Raffelt, Self-induced neutrino flavor conversion without flavor mixing, J. Cosmol. Astropart. Phys. 03 (2016) 042.

[11] I. Izaguirre, G. Raffelt, and I. Tamborra, Fast Pairwise Conversion of Supernova Neutrinos: A Dispersion-Relation Approach, Phys. Rev. Lett. 118, 021101 (2017).

[12] M.-R. Wu and I. Tamborra, Fast neutrino conversions: Ubiquitous in compact binary merger remnants, Phys. Rev. D 95, 103007 (2017).

[13] F. Capozzi, B. Dasgupta, E. Lisi, A. Marrone, and A. Mirizzi, Fast flavor conversions of supernova neutrinos: Classifying instabilities via dispersion relations, Phys. Rev. D 96, 043016 (2017).
[14] B. Dasgupta, A. Mirizzi, and M. Sen, Fast neutrino flavor conversions near the supernova core with realistic flavordependent angular distributions, J. Cosmol. Astropart. Phys. 02 (2017) 019.

[15] S. Abbar and H. Duan, Fast neutrino flavor conversion: Roles of dense matter and spectrum crossing, Phys. Rev. D 98, 043014 (2018).

[16] B. Dasgupta and M. Sen, Fast neutrino flavor conversion as oscillations in a quartic potential, Phys. Rev. D 97, 023017 (2018).

[17] S. Abbar and M. Cristina Volpe, On fast neutrino flavor conversion modes in the nonlinear regime, Phys. Lett. B 790, 545 (2019).

[18] S. Airen, F. Capozzi, S. Chakraborty, B. Dasgupta, G. Raffelt, and T. Stirner, Normal-mode analysis for collective neutrino oscillations, J. Cosmol. Astropart. Phys. 12 (2018) 019.

[19] S. Abbar, H. Duan, K. Sumiyoshi, T. Takiwaki, and M. C. Volpe, On the occurrence of fast neutrino flavor conversions in multidimensional supernova models, arXiv:1812.06883.

[20] S. Chakraborty, R. Hansen, I. Izaguirre, and G. Raffelt, Collective neutrino flavor conversion: Recent developments, Nucl. Phys. B908, 366 (2016).

[21] C. W. Kim, J. Kim, and W. K. Sze, On the geometrical representation of neutrino oscillations in vacuum and matter, Phys. Rev. D 37, 1072 (1988).

[22] H. Duan, G. M. Fuller, and Y.-Z. Qian, Collective neutrino flavor transformation in supernovae, Phys. Rev. D 74, 123004 (2006).

[23] H. Duan, G. M. Fuller, and Y.-Z. Qian, Neutrino flavor spin waves, arXiv:0808.2046v1.

[24] G. Mangano, A. Mirizzi, and N. Saviano, Damping the neutrino flavor pendulum by breaking homogeneity, Phys. Rev. D 89, 073017 (2014).

[25] H. Duan and S. Shalgar, Flavor instabilities in the neutrino line model, Phys. Lett. B 747, 139 (2015).

[26] S. Chakraborty, R. Sloth Hansen, I. Izaguirre, and G. Raffelt, Self-induced flavor conversion of supernova 
neutrinos on small scales, J. Cosmol. Astropart. Phys. 01 (2016) 028.

[27] A. Mirizzi, G. Mangano, and N. Saviano, Self-induced flavor instabilities of a dense neutrino stream in a twodimensional model, Phys. Rev. D 92, 021702 (2015).

[28] S. Abbar and H. Duan, Neutrino flavor instabilities in a timedependent supernova model, Phys. Lett. B 751, 43 (2015).

[29] B. Dasgupta and A. Mirizzi, Temporal instability enables neutrino flavor conversions deep inside supernovae, Phys. Rev. D 92, 125030 (2015).

[30] H. Duan, Collective neutrino oscillations and spontaneous symmetry breaking, Int. J. Mod. Phys. E 24, 1541008 (2015).

[31] L. Ma, Neutrino flavor conversions in dense media, Ph.D. thesis, University of New Mexico, 2018.

[32] P. A. Sturrock, Kinematics of growing waves, Phys. Rev. 112, 1488 (1958).
[33] R. J. Briggs, Electron-Stream Interaction with Plasmas (MIT Press, Cambridge, MA, 1964).

[34] B. Dasgupta, A. Mirizzi, and M. Sen, Simple method of diagnosing fast flavor conversions of supernova neutrinos, Phys. Rev. D 98, 103001 (2018).

[35] G. Sigl and G. Raffelt, General kinetic description of relativistic mixed neutrinos, Nucl. Phys. B406, 423 (1993).

[36] A. Banerjee, A. Dighe, and G. Raffelt, Linearized flavorstability analysis of dense neutrino streams, Phys. Rev. D 84, 053013 (2011).

[37] G. Raffelt, S. Sarikas, and D. de Sousa Seixas, Axial Symmetry Breaking in Self-Induced Flavor Conversion of Supernova Neutrino Fluxes, Phys. Rev. Lett. 111, 091101 (2013).

[38] H. Duan, Flavor oscillation modes in dense neutrino media, Phys. Rev. D 88, 125008 (2013). 